\title{
Platelet Derived Growth Factor-B and Human Epidermal Growth Factor Receptor-2 Polymorphisms in Gall Bladder Cancer
}

\author{
Kumudesh Mishra ${ }^{1,2}$, Anu Behari ${ }^{1}$, Vinay Kumar Kapoor ${ }^{1}$, M. Salman Khan ${ }^{2}$, \\ Swayam Prakash ${ }^{3}$, Suraksha Agrawal ${ }^{3 *}$
}

\begin{abstract}
Gall bladder cancer (GBC) is a gastro-intestinal cancer with high prevalence among north Indian women. Platelet derived growth factor-B (PDGFB) and human epidermal growth factor receptor-2 (HER2) may play roles in the etiology of GBC through the inflammation-hyperplasia-dysplasia-carcinoma pathway. To study the association of $P D G F B$ and $H E R 2$ polymorphisms with risk of $\mathrm{GBC}, 200$ cases and 300 controls were considered. $P D G F B+286 \mathrm{~A}>\mathrm{G}$ and $+1135 \mathrm{~A}>\mathrm{C}$ polymorphisms were investigated with an amplification refractory mutation system and the HER2 $\mathrm{Ile}^{655}$ Val polymorphism by restriction fragment length polymorphism. Significant risk associations for PDGFB +286 GG $(\mathrm{OR}=5.25)$ and $P D G F B+1135 \mathrm{CC}(\mathrm{OR}=3.19)$ genotypes were observed for GBC. Gender wise stratification revealed susceptibility for recessive models of $P D G F B+1135 \mathrm{~A}>\mathrm{C}(\mathrm{OR}=3.00)$ and HER2 $\mathrm{Il}^{655} \mathrm{Val}(\mathrm{OR}=2.52)$ polymorphisms among female $\mathrm{GBC}$ cases. GBC cases with gall stones were predisposed to homozygous $+286 \mathrm{GG}$ and $+1135 \mathrm{CC}$ genotypes. Significant risk associations were found for $\mathrm{ACIle}(\mathrm{OR}=1.48)$, GAVal $(\mathrm{OR}=1.70)$, GAIle $(\mathrm{OR}=2.00)$ haplotypes with $\mathrm{GBC}$ cases and GCIle haplotype with female GBC cases $(\mathrm{OR}=10.37, \mathrm{P}=<0.0001)$. Pair-wise linkage disequilibrium revealed negative associations among variant alleles. On multi-dimensional reduction analysis, a three factor model revealed significant gene-gene interaction for $P D G F B+286 \mathrm{~A}>\mathrm{G}, P D G F B+1135 \mathrm{~A}>\mathrm{C}$ and HER2 Ile165Val SNPs with GBC. Protein-protein interaction showed significant association of $P D G F B$ and $H E R 2$ with the epidermal growth factor receptor signaling pathway.
\end{abstract}

Keywords: Gallbladder cancer - HER2 - PDGFB - single nucleotide polymorphisms

Asian Pac J Cancer Prev, 16 (14), 5647-5654

\section{Introduction}

Gall bladder cancer (GBC) is an uncommon etiology with late diagnosis, limited treatment options and poor prognosis with overall five year survival rate of less than $10 \%$. Remarkable variation in the incidence of GBC has been reported across the globe with low prevalence in the United States, United Kingdom and western Europe, and higher frequency in central and south America, central and eastern Europe, Japan (Randi et al., 2006) and China (Qu et al.,2012). Chile in south America has one of the highest incidence rates of GBC in world and GBC is the major cause of cancer deaths in females (Randi et al., 2006). It is reported as a disease of elderly females (Hamdani et al., 2012). In context to India, GBC is more prevalent among north Indians in comparison to their southern counterpart. Its incidence is to the tune of nine affected female cases per one lac population in north India (NCRP, 2002). The peculiar geographical and racial variations in its incidence suggest the importance of genetic factors in etiology of GBC. Earlier association of single nucleotide polymorphisms (SNPs) with GBC has been reported (Mishra et al., 2013).

Angiogenesis is a key factor for tumor growth and metastasis. Platelet derived growth factor (PDGF) is a critical mediator of tumor angiogenesis. $P D G F B$ expression may result into uncontrolled replication of neoplastic cells leading to the progression of GBC. $P D G F B$ gene expression has been studied in various cancers e.g. lung cancer, esophageal cancer, gastric cancer, pancreatic cancer, etc (Bravo et al., 1991; Chung et al., 1992; Wong et al., 1994; Yamamoto et al., 1996). However, genetic association of $P D G F B+286 \mathrm{~A}>\mathrm{G}$ and $+1135 \mathrm{~A}>\mathrm{C}$ SNPs have been studied first time in hepatitis C infection case (Ben-Ari et al., 2006).

Human epidermal growth factor receptor-2 (HER2/cerbB-2) is a proto-oncogene member of the epidermal growth factor receptor (EGFR) family. Structural and functional alteration of HER2 has been reported in different steps of carcinogenesis including initiation,

${ }^{1}$ Department of Surgical Gastroenterology, ${ }^{3}$ Department of Medical Genetics, Sanjay Gandhi Postgraduate Institute of Medical Sciences, ${ }^{2}$ Department of Biosciences, Integral University, Lucknow, India *For correspondence: suraksha@sgpgi.ac.in, sur_ ksha_agrawal@yahoo.co.in 
promotion and progression (Marmor et al., 2004). SNP at codon 655 of HER2 gene has been identified which encodes either isoleucine (Ile: ATC) or valine (Val: GTC) in the transmembrane domain-coding region (Papewalis et al., 1991; Xie et al., 2000). It has also been showed that HER2 can be successfully used as a tumor marker for breast cancer (Xie et al., 2000). To assess HER2 association with other cancers like hepato-cellular, gastric, ovarian, bladder, colo-rectal, prostate etc. various studies have been conducted (Nakajima et al., 1999; McKay et al., 2002; Wang et al., 2002; Kuraoka et al., 2003; Quaye et al., 2009; Karaca et al., 2012; Zahir et al., 2014). PDGFB and $H E R 2$ belong to a family of receptor tyrosine kinases that are thought to participate in signal transduction mediating tumor cell proliferation and/or motility (Nakajima et al., 1999) that is why we studied both together.

The present cross sectional study was designed to investigate genetic associations of $P D G F B+286 \mathrm{~A}>\mathrm{G}$ (rs\#1800818), PDGFB +1135A >C (rs\#1800817) and HER2 $\mathrm{Ile}^{655}$ Val (rs\#11362000) polymorphisms and their haplotypes with GBC. Previously no genetic association studies of $P D G F B$ and $H E R 2$ polymorphim have been done in gallbladder cancer.

\section{Materials and Methods}

\section{Ethics Statement}

The study was approved by the Institutional Ethical Committee (IEC) of the Sanjay Gandhi Postgraduate Institute of Medical Sciences (SGPGIMS), Lucknow India. All the participants were provided with and signed written informed consent for the study. The recruitment of subjects was carried out according to norms of the Declaration of Helsinki.

\section{Subjects}

The study included 200 GBC cases of which five cases were excluded due to erroneous data. The demographic characteristics were evaluated based on gender, $119(61 \%)$ females and $76(39 \%)$ males were present in out of 195

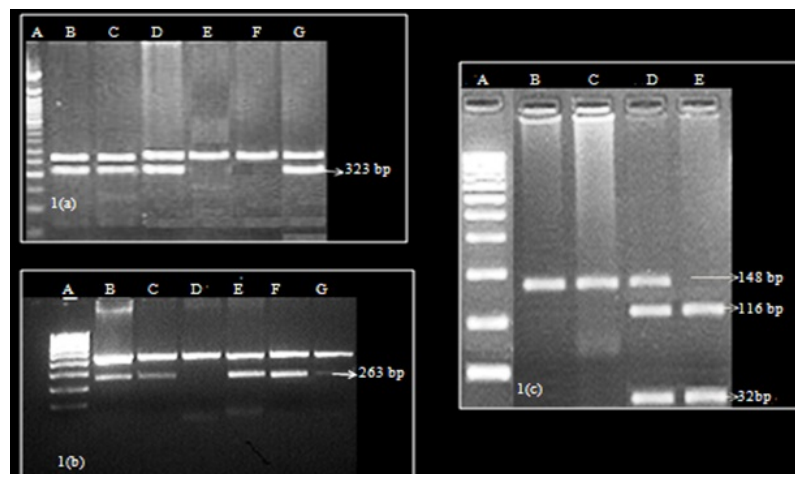

Figure 1. Representing Gel Images Showing: 1(a) PDGFB 286A>G Polymorphism, lane B+C-AG, D+E$A A$ and F+G-GG genotype; 1(b) PDGFB 1135A $>C$ Polymorphism, Lane B+C-AC, D+E-CC and F+G-AA genotype; 1(c) HER2 Ile ${ }^{655}$ Val Polymorphism, Lane B+C-Ile/Ile, D-Val/Val and E-Ile/Val genotype. In lane A-100bp DNA ladder showed in 1 (a) and 1(b) and a 50bp ladder showed in 1(c)
GBC cases. The mean age with standard deviation of GBC cases was $52.7 \pm 11.2$. Gall stone (GS) was present in $122(62.6 \%)$ whereas GS was absent in $73(37.4 \%)$ GBC cases. All the cases were registered in the Department of Surgical Gastroenterology, SGPGIMS, Lucknow, a tertiary care hospital in north India. Gender matched controls, $200(67 \%)$ females and 100 (33\%) males were included in this study, as GBC is predominantly found among females .The mean age with standard deviation of controls was $43.6 \pm 11.0$. Blood samples for controls with no prior history of cancer were collected from two previous studies (Aggarwal et al., 2011; Prakash et al., 2012). The controls had similar ethnic background to that of the cases. GBC was confirmed by fine needle aspiration cytology (FNAC) and/or histopathology. Presence or absence of GS in GBC was confirmed by ultrasonography (US) or at the time of surgery. DNA was extracted from venous blood by using QIAamp DNA Blood Mini Kit (QIAGEN, Valencia, CA, USA).

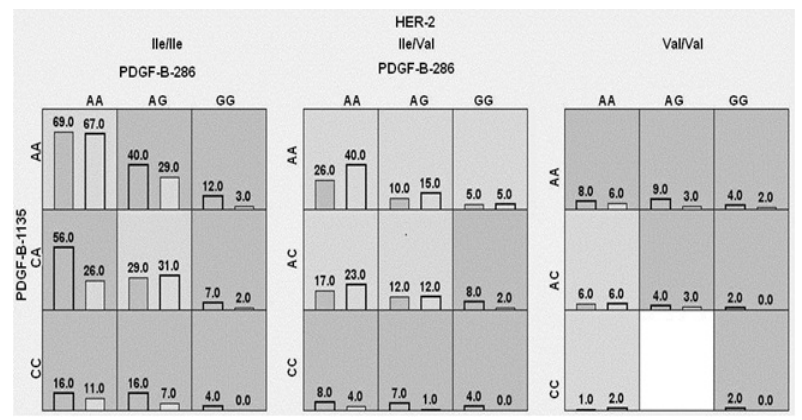

Figure 2. The Best Model in MDR Analyses was Composed of PDGFB +286A > G-PDGFB +1135 A >C-

HER2 Ile655Val GB. In each cell of genotype combination, the left bar represents score for GBC and the right bar for Normal Control. High-risk combinations for GBC are shaded dark grey, while low-risk combinations are shaded light grey

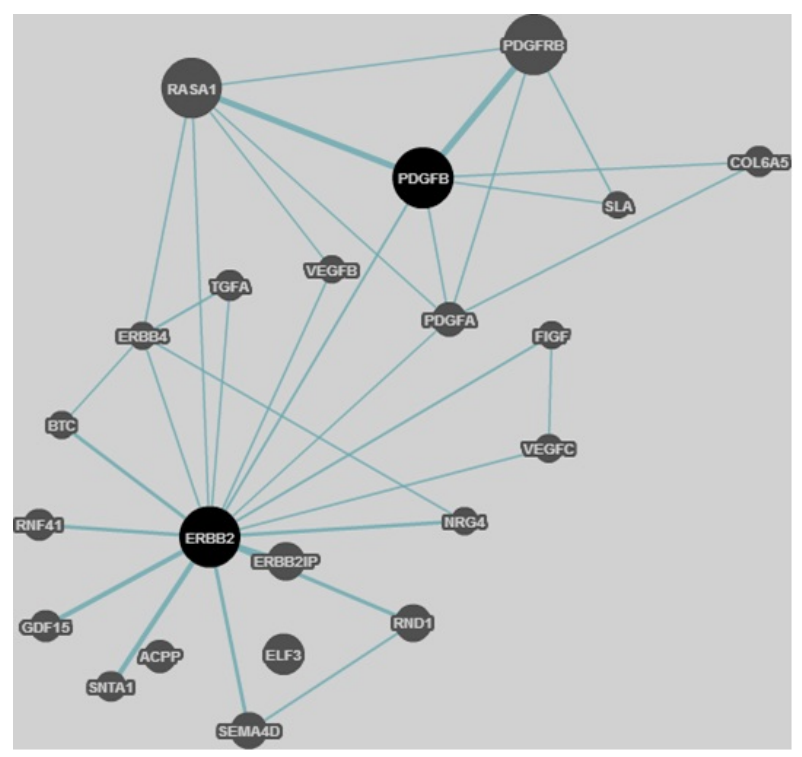

Figure 3. Protein-protein Network Analysis Showing Major Genes Linked with PDGFB-HER2 Associated Biological Pathways. Prominence of genes in the network is evidenced from the weights assigned basing on number of interactions involved with a particular gene 
Platelet Derived Growth Factor-B and Human Epidermal Growth Factor Receptor-2 Polymorphisms and Gall Bladder Cancer

\section{Genotyping}

Amplification of $P D G F B+286 \mathrm{~A}>\mathrm{G}, P D G F B$ $+1135 \mathrm{~A}>\mathrm{C}$ and HER2 $\mathrm{Ile}^{655}$ Val SNPs were carried out in a thermal cycler (Mastercycler gradient; Eppendorf, Hamburg, Germany). Genotyping was done for $P D G F B+286 \mathrm{~A}>\mathrm{G}$ and $+1135 \mathrm{~A}>\mathrm{C}$ polymorphisms by amplification refractory mutation system (ARMS) PCR using a common set of primers as mentioned by (Ben-Ari et al., 2006) and HER2 Ile ${ }^{655}$ Val by restriction fragment length polymorphism (PCR-RFLP) using a common set of primers and PCR conditions as described before (Papewalis et al., 1991; Xie et al., 2000). PCR products of HER2 Ile ${ }^{655}$ Val polymorphisms were digested using BsmAI. The 148 bp PCR product was cut by BsmAI into two fragments of 116 and $32 \mathrm{bp}$ if the Val allele was present, whereas the product of Ile allele was uncut and produced a single fragment of 148 bp (Figure 1). PCR and RFLP products were run by gel electrophoresis on $2 \%$ agarose gel stained with ethidium bromide.

\section{Statistical Analysis}

Statistical power of the study and sample size estimation were carried out using $\mathrm{G}^{*}$ Power version 2 (Heinrich Heine Universitat, Dusseldorf, Germany). Differences in the $P D G F B$ and HER2 genotype, allele frequencies and haplotypes between the study and control groups were analyzed with Fisher exact test and $p$ values $\leq$ 0.05 were considered statistically significant. Odds Ratio (OR) was used to measure strength of association between genotypes, allele frequencies and haplotypes between GBC.Analysis for the genotypes was done under additive, recessive and dominant models of inheritance. Haplotypes were generated using Arlequin software (University of Geneva, Switzerland) and statistical analysis was done using SPSS software version 16 (IBM Corporation, New York, NY USA). The degree of pair wise linkage disequilibrium (LD) was calculated for each pair of SNPs taking into consideration the GBC cases using the SNP Stats program (Catalan Institute of Oncology, IDIBELL, Epidemiology and Cancer Registry L'Hospitalet, Barcelona, Spain) (Sole et al., 2006). Conventional LD was calculated using Lewontin's principle. We have also calculated correlation coefficient (r) using Cramer's V statistic and corresponding $\mathrm{p}$-value for each pair wise LD measure. The multi dimensional reduction (MDR) software version 2.0 beta 8 (Vanderbilt University Medical School, Nashville, TN, USA) (Hahn et al., 2003) was used to identify high-order gene-gene interactions associated with GBC. The nonparametric MDR was used to overcome limitations of logistic regression (i.e., sample size limitations) for the detection and characterization of gene-gene interactions. The MDR results were validated through cross-validation and permutation testing. Proteinprotein interaction network involving $P D G F B$ and $H E R 2$ genes was constructed using Gene MANIA(Warde-Farley et al., 2010) which effectively predicted hypothesis about gene function, analyzed gene lists and prioritized genes for constructing interaction networks.

Table 1. Comparison of PDGFB and HER2 Genotype and Allele Frequency Distribution in GBC with Controls

\begin{tabular}{|c|c|c|c|c|}
\hline Genotype & $\begin{array}{c}\text { Controls }(\mathrm{n}=300) \\
\mathrm{n}(\%)\end{array}$ & $\begin{array}{c}\mathrm{GBC}(\mathrm{n}=195) \\
\mathrm{n}(\%)\end{array}$ & OR $(95 \% \mathrm{CI})$ & P-Value \\
\hline PDGFB+286A>G (rs\#1800818) & & vs Controls & & \\
\hline GG (additive model) & $14(4.5)$ & $37(18.9)$ & $5.25(2.70-10.2)$ & $<0.0001^{\dagger}$ \\
\hline AG (additive model) & $101(33.5)$ & $65(33.4)$ & $0.72(0.46-1.14)$ & 0.1792 \\
\hline AA & $185(62)$ & $93(47.7)$ & & \\
\hline GG vs AA+AG (Recessive model) & & & $4.78(2.51-9.11)$ & $<0.0001^{\dagger}$ \\
\hline AA $v s$ GG+AG (Dominant model) & & & $0.56(0.39-0.81)$ & $0.003 *$ \\
\hline \multicolumn{5}{|l|}{ Allele frequency } \\
\hline $286 \mathrm{G}$ & $129(21.5)$ & $139(35.6)$ & $2.02(1.52-2.68)$ & $<0.0001^{\dagger}$ \\
\hline $286 \mathrm{~A}$ & $471(78.5)$ & $251(64.4)$ & & \\
\hline \multicolumn{5}{|l|}{ PDGFB+1135A $>C($ rs\#1800817) } \\
\hline CC (additive model) & $25(8.5)$ & $39(20)$ & $3.19(1.81-5.63)$ & $<0.0001^{\dagger}$ \\
\hline AC (additive model) & $105(35)$ & $73(37.4)$ & $1.42(0.95-2.11)$ & 0.0846 \\
\hline AA & $170(56.5)$ & $83(42.5)$ & & \\
\hline $\mathrm{CC}$ vs $\mathrm{AA}+\mathrm{AC}$ (Recessive model) & & & $2.75(1.60-4.71)$ & $0.0003^{\dagger}$ \\
\hline AA vs CC+AC (Dominant model) & & & $0.56(0.39-0.81)$ & $0.0024^{*}$ \\
\hline $1135 \mathrm{C}$ & $155(25.8)$ & $151(38.7)$ & $1.81(1.37-2.38)$ & $<0.0001^{\dagger}$ \\
\hline $1135 \mathrm{~A}$ & $445(74.2)$ & $239(61.3)$ & & \\
\hline \multicolumn{5}{|l|}{ HER2 Ile655Val (rs\#1136200) } \\
\hline Val/Val (additive model) & $24(8)$ & $25(12.8)$ & $1.51(0.82-2.77)$ & 0.2119 \\
\hline Ile/Val (additive model) & $102(34)$ & $50(25.6)$ & $0.71(0.47-1.07)$ & 0.1228 \\
\hline Ile/Ile & $174(58)$ & $120(61.5)$ & & \\
\hline $\mathrm{Val} / \mathrm{Val}$ vs Ile/Ile+Ile/Val (Recessive model) & & & $1.69(0.93-3.05)$ & 0.0907 \\
\hline Ile/Ile $v s \mathrm{Val} / \mathrm{Val}+\mathrm{Ile} / \mathrm{Val}$ (Dominant model) & & & $1.15(0.80-1.67)$ & 0.4546 \\
\hline \multicolumn{5}{|l|}{ Allele frequency } \\
\hline Val allele & $150(25)$ & $100(25.6)$ & $1.03(0.77-1.38)$ & 0.8227 \\
\hline Ile allele & $450(75)$ & $290(74.4)$ & & \\
\hline
\end{tabular}

*Statistically significant and risk-protective genotype/allele; ${ }^{\prime}$ Statistically significant and risk-associated genotype/allele; Additive Model: comparing mutant homozygous and heterozygous genotypes individually with wild homozygous type; Recessive Model: comparing mutant homozygous genotype with wild homozygous and heterozygous genotype taken together; Dominant Model: comparing wild homozygous genotype with mutant homozygous and heterozygous genotype taken 


\section{Results}

$P D G F B+286 \mathrm{~A}>\mathrm{G}$ (p-value $=0.9533), P D G F B$ $+1135 \mathrm{~A}>\mathrm{C}(\mathrm{p}-\mathrm{v}$ alue $=0.1690)$ and HER2 $\mathrm{Ile}^{655} \mathrm{Val}$ ( $p$-value $=0.1803$ ) were in Hardy-Weinberg equilibrium at one degree of freedom among controls. This indicated no genotyping errors, inbreeding, genetic drift, and mutation or population substructure. Significant deviations from the expected proportions of homozygote and heterozygote classes among patients may be due to association with the disease allele. Significant statistical power was obtained against GBC and control group combinations (0.99) which justified sample size for the present study.

\section{Genotype and allele frequency}

Genotype and allele frequencies of GBC compared with controls are shown in Table 1. Homozygous GG genotype and $\mathrm{G}$ allele along with the recessive model of $P D G F B+286 \mathrm{~A}>\mathrm{G}$ SNP were found to be risk associated with GBC. CC genotype and recessive model of $P D G F B$ $+1135 \mathrm{~A}>\mathrm{C}$ polymorphism revealed susceptibility whereas dominant model showed protective association with GBC. However, no significant association was found for HER2 $\mathrm{Ile}^{655}$ Val SNP with GBC.

Gender-wise comparison of genotype and allele frequencies of GBC with controls is given in Table 2. Homozygous GG genotype and $\mathrm{G}$ allele of $P D G F B+286$
$\mathrm{A}>\mathrm{G}$ along with $\mathrm{CC}$ genotype and $\mathrm{C}$ allele of $P D G F B$ $+1135 \mathrm{~A}>\mathrm{C}$ SNPs were observed to be risk associated in both male and female GBC cases. Recessive models of $P D G F B+286 \mathrm{~A}>\mathrm{G}, P D G F B+1135 \mathrm{~A}>\mathrm{G}$ and HER2 $\mathrm{Ile}^{655} \mathrm{Val}$ SNPs also showed predisposing associations in female GBC cases. Similarly homozygous Val/ Val genotype of HER2 Ile ${ }^{655} \mathrm{Val}$ SNP also revealed susceptibility with female cases.

\section{Comparison of $G B C$ with and without gall stone (GS)}

GS was observed among $62.6 \%$ of GBC cases in our study. GBC cases were categorized into GBC with and without GS and were compared with normal controls (Table 3). Risk association among GBC cases with and without GS for recessive models of $P D G F B+286 \mathrm{~A}>\mathrm{G}$ and $P D G F B+1135 \mathrm{~A}>\mathrm{C}$ SNPs were evident. Similarly both GBC with and without GS cases were predisposed to GG genotype and $\mathrm{G}$ allele of $P D G F B+286 \mathrm{~A}>\mathrm{G}$ polymorphism along with $\mathrm{CC}$ genotype and $\mathrm{C}$ allele of $P D G F B+1135$ SNP. Heterozygous Ile/Val genotype of HER2 $\mathrm{Ile}^{655} \mathrm{Val}$ polymorphism showed risk protective association in GBC with GS whereas recessive model was risk associated in GBC without GS.

\section{Haplotype analysis}

Seven haplotypes (ACIle, GAVal, AAIle, GAIle, GCIle, AAVal and ACVal) were found to be common

Table 2. Gender wise Comparison of PDGFB and HER2 Genotype and Allele Frequency Distribution in GBC with Controls

\begin{tabular}{|c|c|c|c|c|c|c|c|c|}
\hline \multirow[t]{3}{*}{ Genotype } & \multicolumn{4}{|c|}{ Male } & \multicolumn{4}{|c|}{ Female } \\
\hline & $\begin{array}{l}\text { Control } \\
(n=100)\end{array}$ & $\begin{array}{c}\text { GBC } \\
(n=76)\end{array}$ & OR $(95 \% \mathrm{CI})$ & P-Value & $\begin{array}{l}\text { Control } \\
(n=200)\end{array}$ & $\begin{array}{c}\text { GBC } \\
(n=119)\end{array}$ & OR $(95 \%$ CI $)$ & P-Value \\
\hline & $\mathrm{n}(\%)$ & $\mathrm{n}(\%)$ & & & $\mathrm{n}(\%)$ & $\mathrm{n}(\%)$ & & \\
\hline \multicolumn{3}{|l|}{ PDGFB+286A>G (rs\#1800818) } & GBC vs Controls & & & & & \\
\hline GG (additive model) & $5(5)$ & $18(23.8)$ & $7.57(2.55-22.4)$ & $<0.0001 \dagger$ & $9(4.5)$ & $19(15.9)$ & $4.09(1.75-9.55$ & $0.0014 \dagger$ \\
\hline AG (additive model) & $34(34)$ & $29(38.1)$ & $1.79(0.92-3.48)$ & 0.0927 & $67(33.5)$ & $36(30.2)$ & $1.04(0.62-1.72)$ & 0.8978 \\
\hline $\mathrm{AA}$ & $61(61)$ & $29(38.1)$ & & & $124(62)$ & $64(53.9)$ & & \\
\hline \multicolumn{3}{|c|}{ GG vs AA+AG (Recessive model) } & $5.89(2.07-16.74)$ & $0.0005 \dagger$ & & & $4.03(1.75-9.24)$ & $0.0008 \dagger$ \\
\hline \multicolumn{3}{|c|}{ AA vs GG+AG (Dominant model) } & $0.39(0.21-0.72)$ & $0.0037 *$ & & & $0.71(0.45-1.13)$ & 0.1593 \\
\hline \multicolumn{9}{|c|}{ Allele frequency } \\
\hline $286 \mathrm{G}$ & $44(22)$ & $65(42.8)$ & $2.64(1.66-4.21)$ & $<0.0001 \dagger$ & $85(21.2)$ & $74(31.1)$ & $1.62(1.16-2.40)$ & $0.0061 \dagger$ \\
\hline $286 \mathrm{~A}$ & $156(78)$ & $87(57.2)$ & & & $315(78.8)$ & $164(68.9)$ & & \\
\hline \multicolumn{9}{|l|}{$\mathrm{PDGFB}+1135 \mathrm{~A}>\mathrm{C}(\mathrm{rs} \# 1800817)$} \\
\hline CC (additive model) & $8(8)$ & $13(17.1)$ & $2.98(1.11-7.99)$ & $0.0458 \dagger$ & $17(8.5)$ & $26(21.8)$ & $3.32(1.66-6.65)$ & $0.0007 \dagger$ \\
\hline AC (additive model) & $35(35)$ & $32(42.1)$ & $1.68(0.87-3.21)$ & 0.1382 & $70(35)$ & $41(34.4)$ & $1.27(0.76-2.11)$ & 0.3657 \\
\hline AA & $57(57)$ & $31(40.8)$ & & & $113(56.5)$ & $52(43.6)$ & & \\
\hline \multicolumn{3}{|c|}{$\mathrm{CC}$ vs $\mathrm{AA}+\mathrm{AC}$ (Recessive model) } & $2.37(0.92-6.06)$ & 0.0986 & & & $3.00(1.55-5.82)$ & $0.0011 \dagger$ \\
\hline \multicolumn{3}{|c|}{ AA vs $\mathrm{CC}+\mathrm{AC}$ (Dominant model) } & $0.51(0.28-0.95)$ & $0.0476^{*}$ & & & $0.59(0.37-0.94)$ & $0.0284 *$ \\
\hline \multicolumn{9}{|l|}{ Allele frequency } \\
\hline $1135 \mathrm{C}^{2}$ & $51(25.5)$ & $58(38.2)$ & $1.80(1.14-2.84)$ & $0.0144 \dagger$ & $104(26)$ & $93(39.1)$ & $1.82(1.29-2.57)$ & $0.0007 \dagger$ \\
\hline $1135 \mathrm{~A}$ & $149(74.5)$ & $94(68.8)$ & & & $296(74)$ & $145(60.9)$ & & \\
\hline \multicolumn{9}{|l|}{ HER2 Ile655Val (rs\#1136200) } \\
\hline Val/Val (additive model) & $8(8)$ & $6(7.9)$ & $0.85(0.27-2.62)$ & 1.0 & $14(7)$ & $19(15.9)$ & $2.31(1.09-4.92)$ & $0.0335 \dagger$ \\
\hline Ile/Val (additive model) & $34(34)$ & $19(25)$ & $0.63(0.32-1.24)$ & 0.2369 & $68(34)$ & $31(26.2)$ & $0.77(0.46-1.30)$ & 0.3645 \\
\hline Ile/Ile & $58(58)$ & $51(67.1)$ & & & $118(59)$ & $69(57.9)$ & & \\
\hline \multicolumn{3}{|c|}{ Val/Val vs Ile/Ile+Ile/Val (Recessive model) } & $0.98(0.32-2.97)$ & 1.0 & & & $2.52(1.21-5.24)$ & $0.0135 \dagger$ \\
\hline \multicolumn{3}{|c|}{ Ile/Ile vs Val/Val+Ile/Val (Dominant model) } & $1.47(0.79-2.75)$ & 0.2728 & & & $0.95(0.60-1.520$ & 0.9066 \\
\hline \multicolumn{9}{|l|}{ Allele frequency } \\
\hline Val allele & $50(25)$ & $31(20.4)$ & $0.76(0.46-1.27)$ & 0.371 & $96(24)$ & $69(28.9)$ & $1.29(0.90-1.85)$ & 0.1905 \\
\hline Ile allele & $150(75)$ & $121(79.6)$ & & & $304(76)$ & $169(71.1)$ & & \\
\hline
\end{tabular}

*Statistically significant and risk-protective genotype/allele; ${ }^{\dagger}$ Statistically significant and risk-associated genotype/allele; Additive Model: comparing mutant homozygous and heterozygous genotypes individually with wild homozygous type; Recessive Model: comparing mutant homozygous genotype with wild homozygous and heterozygous genotype taken together; Dominant Model: comparing wild homozygous genotype with mutant homozygous and heterozygous genotype taken 
Platelet Derived Growth Factor-B and Human Epidermal Growth Factor Receptor-2 Polymorphisms and Gall Bladder Cancer

Table 3. Comparison of PDGF-B and HER-2 Genotype Frequency Distribution in GBC (with and without GS) with Controls

\begin{tabular}{|c|c|c|c|c|c|c|c|c|}
\hline \multirow[b]{3}{*}{$\mathrm{PDGFB}+286 \mathrm{~A}>\mathrm{G}(\mathrm{rs} \# 18008$} & \multirow{2}{*}{$\begin{array}{l}\text { Control } \\
(\mathrm{n}=300) \\
\mathrm{n}(\%)\end{array}$} & \multicolumn{3}{|c|}{ GBC with GS $(n=122)$} & \multirow{2}{*}{$\begin{array}{c}\begin{array}{c}\text { Control } \\
(\mathrm{n}=300)\end{array} \\
\mathrm{n}(\%)\end{array}$} & \multicolumn{3}{|c|}{ GBC without GS $(n=73)$} \\
\hline & & $\mathrm{n}(\%)$ & & & & $\mathrm{n}(\%)$ & & \\
\hline & & & & & & & & \\
\hline GG (additive model) & $14(4.5)$ & $23(18.9)$ & $5.52(2.66-11.4)$ & $<0.0001 \dagger$ & $14(4.5)$ & $14(19.2)$ & $4.68(2.14-11.0)$ & $0.0002 \dagger$ \\
\hline AG (additive model) & $101(33.5)$ & $44(36.0)$ & $1.46(0.92-2.33)$ & 0.1182 & $101(33.5)$ & $21(28.8)$ & $1.01(0.56-1.81)$ & 1.0 \\
\hline AA & $185(62)$ & $55(45.1)$ & & & $185(62)$ & $38(52.0)$ & & \\
\hline \multicolumn{2}{|c|}{ GG vs AA+AG (Recessive model) } & & $4.74(2.35-9.58)$ & $<0.0001 \dagger$ & & & $4.84(2.19-10.7)$ & $0.0002 \dagger$ \\
\hline $\mathrm{AA}$ vs $\mathrm{GG}+\mathrm{AG}$ (Domin & del) & & $3-0.78)$ & 0.0023 & & & .12) & 0.1445 \\
\hline \multicolumn{9}{|l|}{ Allele frequency } \\
\hline $286 \mathrm{G}$ & $129(21.5)$ & $90(36.9)$ & $2.13(1.54-2.95)$ & $<0.0001 \dagger$ & $129(21.5)$ & $49(33.6)$ & $1.84(1.24-2.73)$ & $0.0033 \dagger$ \\
\hline $286 \mathrm{~A}$ & $471(78.5)$ & $154(63.1)$ & & & $471(78.5)$ & $97(66.4)$ & & \\
\hline \multicolumn{9}{|l|}{$\mathrm{PDGFB}+1135 \mathrm{~A}>\mathrm{C}(\mathrm{rs} \# 18 \mathrm{C}$} \\
\hline $\mathrm{CC}$ (additive $\mathrm{m}$ & $25(8.5)$ & $23(18.9)$ & 2.89 & $0.0014 \dagger$ & $25(8.5)$ & $16(21.9)$ & .87) & $0.0007 \dagger$ \\
\hline $\mathrm{AC}(\mathrm{adc}$ & $105(35)$ & $45(36.9)$ & $1.34(0.84-2.14)$ & 0.2321 & $105(35)$ & $28(38.4)$ & $1.56(0.88-2.77)$ & 0.1387 \\
\hline AA & $170(56.5)$ & $54(44.2)$ & & & $170(56.5)$ & $29(39.7)$ & & \\
\hline $\mathrm{CC}$ vs $\mathrm{AA}+\mathrm{AC}$ (Recess & del) & & $2.55(1.38-4.70)$ & $0.0036 \dagger$ & & & $-6.15)$ & $0.0027 \dagger$ \\
\hline AA vs CC+AC (Dor & del) & & 0.60 & $0.0239^{*}$ & & & .84) & $0.0126^{*}$ \\
\hline \multicolumn{9}{|l|}{ Allele frequency } \\
\hline $1135 \mathrm{C}$ & $155(25.8)$ & $91(37.3)$ & $1.70(1.24-2.34)$ & $0.0011 \dagger$ & $155(25.8)$ & $60(41.1)$ & $2.00(1.37-2.92)$ & $0.0005 \dagger$ \\
\hline $1135 \mathrm{~A}$ & $445(74.2)$ & $153(62.7)$ & & & $445(74.2)$ & $86(58.9)$ & & \\
\hline \multicolumn{9}{|l|}{ HER2 Ile655Val (rs\#1136200) } \\
\hline Val/Val (additive model & $24(8)$ & $13(10.6)$ & 1.17 & 0.7069 & $24(8)$ & $12(16.4)$ & .71) & 0.0729 \\
\hline Ile/Val (additive model) & $102(34)$ & $29(23.8)$ & $0.61(0.37-1.01)$ & $0.0569 *$ & $102(34)$ & $21(28.8)$ & $0.89(0.50-1.60)$ & 0.77 \\
\hline Ile/Ile & $174(58)$ & $80(65.6)$ & & & $174(58)$ & $40(54.8)$ & & \\
\hline Val/Val vs Ile/Ile+Ile & ssive model) & & 67-2.79) & 0.4475 & & & $-4.77)$ & $0.0439 \dagger$ \\
\hline Ile/Ile vs $\mathrm{Val} / \mathrm{Val}+\mathrm{Ile}$ & inant model) & & $1.37(0.88-2.13)$ & 0.1557 & & & $0.87(0.52-1.46)$ & 0.6929 \\
\hline \multicolumn{9}{|l|}{ Allele frequency } \\
\hline Val allele & $150(25)$ & $55(22.5)$ & $0.87(0.61-1.24)$ & 0.4795 & $150(25)$ & $45(30.8)$ & $1.33(0.89-1.98)$ & 0.172 \\
\hline Ile allele & $450(75)$ & $189(77.5)$ & & & $450(75)$ & $101(69.2)$ & & \\
\hline
\end{tabular}

*Statistically significant and risk-protective genotype/allele; $\uparrow$ Statistically significant and risk-associated genotype/allele; Additive Model: comparing mutant homozygous and heterozygous genotypes individually with wild homozygous type; Recessive Model: comparing mutant homozygous genotype with wild homozygous and heterozygous genotype taken together; Dominant Model: comparing wild homozygous genotype with mutant homozygous and heterozygous genotype taken

among GBC and normal control groups. Three haplotypes i.e. ACIle $(\mathrm{OR}=1.48$, $\mathrm{p}$-value $=0.0005), \mathrm{GAVal}(\mathrm{OR}=1.70$, $\mathrm{p}$-value $=0.0444)$ and GAIle $(\mathrm{OR}=2.00, \mathrm{p}$-value $=0.004)$ were predisposed to GBC while AAIle $(\mathrm{OR}=0.14$, $\mathrm{p}$-value $=<0.0001)$ and AAVal $(\mathrm{OR}=0.53$, $\mathrm{p}$-value $=0.0051)$ showed protective association. In gender wise stratification of $\mathrm{GBC}$ cases, GAVal $(\mathrm{OR}=2.82$, $\mathrm{p}$-value $=0.0255)$, AAIle $(\mathrm{OR}=2.07, \mathrm{p}$-value $=0.0349)$, GAIle $(\mathrm{OR}=2.90$, $\mathrm{p}$-value $=0.0025)$, and AAVal $(\mathrm{OR}=2.82, \mathrm{p}$-value $=0.0255)$ haplotypes showed risk association in male GBC cases. GCIle $(\mathrm{OR}=10.3$, $\mathrm{p}$-value $=<0.0001)$ haplotype showed risk association while AAIle $(\mathrm{OR}=0.39, \mathrm{p}$-value $=0.0008)$ haplotypes showed protective associations among females GBC cases.

\section{Linkage disequilibrium (LD) analysis}

The pair wise analysis revealed weak and negative LD in our study. Significantly weak LD were noted among all the variant allele pairs like $P D G F B+286 \mathrm{G} /+1135 \mathrm{C}$ $(\mathrm{D}=0.12, \mathrm{r}=0.53), P D G F B+286 \mathrm{G} /$ HER2 Valine $(\mathrm{D}=0$. $11, \mathrm{r}=0.48)$ and $P D G F B+1135 \mathrm{C} /$ HER2 Valine $(\mathrm{D}=0.12$, $\mathrm{r}=0.51)$.

\section{Gene-gene interaction analysis}

To carry out MDR analysis GBC cases and normal controls were considered. Results so obtained were validated through cross-validation and permutation testing. Results revealed best one-factor model for $P D G F B$ $+1135 \mathrm{~A}>\mathrm{G}$ (testing accuracy $=0.5015$, cross-validation consistency $(\mathrm{CVC})=6 / 10$, permutation $(\mathrm{P})=0.023)$, twofactor model for $P D G F B+286 \mathrm{~A}>\mathrm{G}$ and $P D G F B+1135$ $\mathrm{A}>\mathrm{C}$ (testing accuracy $=0.5191, \mathrm{CVC}=7 / 10, \mathrm{P}=0.0001$ ). The three-factor model revealed significant gene-gene interaction for three SNPs namely $P D G F B+286 \mathrm{~A}>\mathrm{G}$, $P D G F B+1135 \mathrm{~A}>\mathrm{G}$ and HER2 $\mathrm{Ile}^{655} \mathrm{Val}$ with GBC (testing accuracy $=0.5484, \mathrm{CVC}=10 / 10, \mathrm{P} \leq 0.0001$ ). There were sixteen genotype combinations involving these SNPs which have showed risk association for GBC (Figure 2).

The MDR analysis revealed presence of higher frequencies and mutant homozygous or heterozygous genotype combinations of $P D G F B$ and HER2 alleles (Ile/Val-CC-AG $=12 \%$, Ile/Ile-AA-GG=6.1\%, Ile/Val$\mathrm{AC}-\mathrm{GG}=5.8 \%$, Ile/Ile-AC-GG=5.1\%, and Val/Val-AA$\mathrm{AG}=4.6 \%$ ) increase the risk of GBC.

\section{Impact of PDGFB and HER2 on biological pathway}

Protein-protein interaction datasets were generated by taking into account the genes that interact in close proximity with $P D G F B$ and HER2 (Figure 3). Genes falling in the network were assigned with weights depending on their association with PDGFB and HER2 in various biological pathways. Network analysis revealed 111 prominent biological functions to be associated with $P D G F B, H E R 2$ associated pathways of which most prominent ones are: epidermal growth factor receptor signaling pathway $(\mathrm{P}=1.62 \mathrm{E}-12)$, ERBB signaling pathway $(\mathrm{P}=1.62 \mathrm{E}-12)$, phosphatidylinositol-mediated signaling $(\mathrm{P}=6.52 \mathrm{E}-10)$, inositol lipid-mediated signaling 
Kumudesh Mishra et al

$(\mathrm{P}=6.52 \mathrm{E}-10)$ and fibroblast growth factor receptor signaling pathway $(\mathrm{P}=2.82 \mathrm{E}-8)$.

\section{Discussion}

Autocrine pathway involving $P D G F B / P D G F R$ signaling establishes self-sufficiency in growth for cancer cells. PDGF is frequently produced by tumor cells and affects tumor growth and dissemination by different means. Amplification and over expression of $P D G F B$ and $H E R 2$ are usually involved in the growth, progression and metastasis of established tumors. Genetically prominent associations of the $P D G F B$ gene polymorphisms with GBC were found in the present study. Mutant homozygous genotypes $+286 \mathrm{GG}(\mathrm{OR}=5.25)$ and $+1135 \mathrm{CC}(\mathrm{OR}=3.19)$ along with mutant alleles $+286 \mathrm{G}(\mathrm{OR}=2.02)$ and $+1135 \mathrm{C}$ $(\mathrm{OR}=1.81)$ of $P D G F B$ showed increased risk association with GBC.

PDGF belongs to the PDGF/VEGF (vascular endothelial growth factor) family. An earlier study by our group has shown association of VEGF SNPs with GBC (Mishra et al., 2013). Since PDGF belongs to the same family as VEGF; it is justified to investigate possible clinical significance of SNPs in related PDGF system. There is no existing literature available to correlate our results with other genetic association studies of PDGF SNPs in the milieu of GBC. However, some reports have suggested the association of $P D G F B$ markers in hepatitis C (Ben-Ari et al., 2006) and chronic pancreatitis (Muddana et al., 2010). The AA genotype of +1135 A $>C$ SNP was in predominance among patients with recurrent HCV infection; however, no association was observed for +286 A $>$ G SNP with the studied liver etiology (BenAri et al., 2006). Muddana et al, in a study on recurrent acute pancreatitis (RAP) and chronic pancreatitis (CP) patients found no difference in genotypic frequencies of $+286 \mathrm{~A}>\mathrm{G}$ and $+1135 \mathrm{~A}>\mathrm{C}$ SNPs among RAP, CP and controls (Muddana et al., 2010). A study showed coexpression of $P D G F B$ and VEGFR-3 to be associated with lymph node metastasis and poor survival in non squamous cell lung cancer (Donnem et al., 2010). Another similar study reported the prognostic significance of $P D G F B \mathrm{~B}$ expression in esophageal squamous cell carcinoma, suggesting a key role in lymphangiogenesis and tumor growth (Matsumoto et al., 2007). PDGFs and PDGFRs not only promote angiogenesis and direct tumor cell growth but also play an important role in lymphangiogenesis (Cao et al., 2005). GBC is a highly metastatic disease and lymph node metastasis is very common. PDGF may therefore, be playing a role. GBC is associated with GS in majority (60-90\%) of cases. GS causes inflammation of the gallbladder in the form of chronic cholecystitis (CC). The combination of GS and cholecystitis increases the risk of GBC (Hsing et al., 2007). In our study $62.5 \%$ of GBC cases had GS and we found significantly increased risk of the genotypes and alleles of $P D G F B+286 \mathrm{~A}>\mathrm{G}$ $(\mathrm{OR}=5.52$ and $\mathrm{OR}=4.68)$ and $+1135 \mathrm{AA}>\mathrm{C}(\mathrm{OR}=2.89$ and $\mathrm{OR}=3.75$ ) in $\mathrm{GBC}$ with and without GS. This strengthens the fact that $P D G F B$ may play a role in the etiology of GBC through the inflammation-hyperplasia-dysplasiacarcinoma pathway.
From the protein-protein interaction analysis we have observed prominent associations of PDGFB and HER2 genes with epidermal growth factor receptor and ERBB signaling pathways which have well evidenced role in the causation of GBC (Li et al., 2014). Approximately onethird of all human cancers exploit deregulated signaling by the ERBB family for growth, survival and other functions toward tumor perpetuation. The ERBB signaling pathway that includes $H E R 2$ and its downstream genes has been reportedly the most extensive mutated pathway affecting nearly $36.8 \%$ of GBC cases in a Chinese cohort (Li et al., 2014). Significance for the fibroblast growth factor receptor signaling pathway was also observed in the GBC. The fibroblast growth factor activates a signaling pathway that positively regulates the PDGF receptors in oligodendritic progenitor cells which enhances the angiogenesis process leading to progression of different cancers.

Alteration of HER2 encodes the receptor tyrosinekinase which has been implicated in carcinogenesis and is frequently observed in a variety of tumors. We have also found HER2 to be significantly associated with positive regulation of protein tyrosinekinase activity among GBC. A Japanese study of 234 gastric cancer patients and 287 control subjects showed that the frequency of Ile/Val and Val/Val genotypes were significantly higher in patients than in controls $(\mathrm{p}=0.005$ and 0.033 , respectively). Val/Val genotype revealed a significantly higher risk $(\mathrm{OR}=3.25)$ compared to Ile/Ile genotype. This study concluded probable association of HER2 SNP with risk for development of gastric cancer and may act as a predictor for gastric cancer (Kuraoka et al., 2003). In our study, frequency of Val/Val genotype was higher in GBC (12.8\%) than controls (8\%) but we did not find any significant association with HER2 SNP in GBC. (McKay et al., 2002) also did not find any significant association with HER2 SNPs when 249 colorectal cancer patients were compared with 257 normal controls subjects. They found same frequencies for Ile allele (80\%) and Val allele (20\%) in colorectal cancer and controls and suggested that HER2 is not a prognostic marker for colorectal cancer. Kara et al also found no evidence of over-expression of HER 2 on 34 colorectal cancer cases (Kara et al., 2012; Nakazava et al., 2005) studied amplification and over-expression of HER2 in 221 biliary tract carcinomas (BTC), of which 89 were GBC, 28 intrahepatic bile duct cancer, 78 extrahepatic bile duct cancer, and 26 ampulla of Vater cases. Overexpression of HER2 was found in $15.7 \%$ GBC patients which was higher than other BTC patients along with $79 \%$ HER2 gene amplification. HER2 deregulation was also observed to be a significant genetic event leading to non-small cell lung cancer (Panagiotou et al., 2012). It has also been suggested as a potential prognostic marker for targeted therapy of gastric cancer (Rakhshani et al., 2014). An immunohistochemical study at our center by (Kumari et al., 2012) reported $80 \%$ over-expression of HER2 in GBC. These results suggested that HER 2 overexpression/ amplification plays an important role in carcinogenesis.

Upon performing pair-wise linkage disequilibrium we have found significantly weak or negative $D$ values for the studied $P D G F B$ and HER 2 SNPs. This strengthens the fact 
Platelet Derived Growth Factor-B and Human Epidermal Growth Factor Receptor-2 Polymorphisms and Gall Bladder Cancer

that these SNPs do not lie in LD (Ben-Ari et al., 2006).

Gender wise comparison revealed susceptibility for the $P D G F B$ and $H E R 2$ SNPs among female GBC cases. GBC is very common in females in north India; in our study $61 \%$ GBC cases were females. Recessive model of $P D G F B$ $+1135 \mathrm{~A}>\mathrm{C}$ SNP was found significantly risk associated $(\mathrm{OR}=3.00)$ in females with GBC. Val/Val genotype and recessive model of HER2 $\mathrm{Ile}^{655} \mathrm{Val}$ polymorphisms was also found to be risk associated $(\mathrm{OR}=2.31$ and $\mathrm{OR}=2.52)$ in females with GBC. HER2 is an established diagnostic marker in breast cancer and it was thus justified to study this polymorphism. The haplotype analysis revealed three haplotypes i.e. ACIle (OR=1.48, $\mathrm{P}=0.0005)$, GAVal $(\mathrm{OR}=1.70, \mathrm{P}=0.0444)$ and GAIle $(\mathrm{OR}=2.00, \mathrm{P}=0.004)$ to be risk associated with GBC as compared to controls while GCIle haplotype was predisposed among female GBC cases $(\mathrm{OR}=10.37$ and $\mathrm{P}=<0.0001)$ on gender-wise stratification. There is no study to compare haplotypes in GBC. This is, thus, a very important finding but our results need further confirmation on a larger cohort. The three-factor model, involving $P D G F B+286 \mathrm{~A}>\mathrm{G}, P D G F B$ $+1135 \mathrm{~A}>\mathrm{C}$ and HER2 Ile $>$ Val showed significant genegene interactions in GBC. Thirteen high-risk combinations involving $P D G F B+286 \mathrm{~A}>\mathrm{G}, P D G F B+1135 \mathrm{~A}>\mathrm{C}$ and HER2 Ile $>$ Val SNPs showed higher frequency in GBC cases as compared to controls which signifies the probable role of the studied SNPs with GBC.

Our study suggests that $P D G F B+286 \mathrm{~A}>\mathrm{G}$ and $P D G F B+1135 \mathrm{~A}>\mathrm{C}$ SNPs may be susceptible markers for GBC. However, this needs further validation on larger and diverse populations along with gene expression analysis of $P D G F B$ and HER 2 genes. At present, the study cannot be used for disease prediction or diagnosis. However, it may be used for screening of patients with GS to predict their risk for developing GBC.

\section{Acknowledgements}

Mr. Kumudesh Mishra is thankful to the Indian Council of Medical Research (ICMR), New Delhi, India for a Senior Research Fellowship.

\section{References}

Aggarwal S, Parveen F, Faridi RM, et al (2011). Vascular endothelial growth factor gene polymorphisms in North Indian patients with recurrent miscarriages. Reprod Biomed Online, 22, 59-64.

Ben-Ari Z, Tambur AR, Pappo O, et al (2006). Platelet-derived growth factor gene polymorphism in recurrent hepatitis C infection after liver transplantati don. Transplantat, 81, 392-7.

Bravo M, Vasquez R, Rubio H, et al (1991). Production of platelet-derived growth factor by human lung cancer. Respir Med, 85, 479-85.

Cao Y (2005). Opinion: emerging mechanisms of tumour lymphangiogenesis and lymphatic metastasis. Nat Rev Cancer, 5, 735-43.

Chung CK, Antoniades HN (1992). Expression of c-sis/plateletderived growth factor B, insulin-like growth factor I, and transforming growth factor alpha messenger RNAs and their respective receptor messenger RNAs in primary human gastric carcinomas: in vivo studies with in situ hybridization and immunocytochemistry. Cancer Res, 52, 3453-9.

Donnem T, Al-Saad S, Al-Shibli K, Busund LT, Bremnes RM (2010). Co-expression of $P D G F B$ and VEGFR-3 strongly correlates with lymph node metastasis and poor survival in non-small-cell lung cancer. Ann Oncol, 21, 223-31.

Hahn LW, Ritchie MD, Moore JH (2003). Multifactor dimensionality reduction software for detecting gene-gene and gene-environment interactions. Bioinformatics, 19, 376-82.

Hamdani NH, Qadri SK, Aggarwalla R, et al (2012). Clinicopathological study of gall bladder carcinoma with special reference to gallstones: our 8-year experience from Eastern India. Asian Pac J Cancer Prev, 13, 5613-7.

Hsing AW, Gao YT, Han TQ, et al (2007). Gallstones and the risk of biliary tract cancer: a population-based study. $\mathrm{Br} \mathrm{J}$ Cancer, 97, 1577-82.

Kara O, Duman BB, Kara B, et al (2012). Analysis of PTEN, VEGF, HER2 and P53 status in determining colorectal cancerbenefit from bevacizumab therapy. Asian Pac J Cancer Prev, 13, 6397-401.

Karaca H, Deniz K, Berk V, Inanc M, Ozkan M (2012). Association of human epidermal growth factor receptor-2 expression and clinicopathological findings in patients with colorectal cancer. Asian Pac J Cancer Prev, 13, 6221-5.

Kumari N, Kapoor VK, Krishnani N, Kumar K, Baitha DK (2012). Role of C-erbB2 expression in gallbladder cancer. Indian J Pathol Microbiol, 55, 75-9.

Kuraoka K, Matsumura S, Hamai Y, et al (2003). A single nucleotide polymorphism in the transmembrane domain coding region of HER2 is associated with development and malignant phenotype of gastric cancer. Int J Cancer, 107, 593-6.

Li M, Zhang Z, Li X, et al (2014). Whole exome and targeted gene sequencing of gall bladder carcinoma identifies recurrent mutations in the ErBb pathway. Nature Genetics, 46, 872-6.

Marmor MD, Skaria KB, Yarden Y (2004). Signal transduction and oncogenesis by ErbB/HER receptors. Int J Radiat Oncol Biol Phys, 58, 903-13.

Matsumoto S, Yamada Y, Narikiyo M, et al (2007). Prognostic significance of platelet-derived growth factor-BB expression in human esophageal squamous cell carcinomas. Anticancer Res, 27, 2409-14.

McKay JA, Loane JF, Ross VG, et al (2002). C-erbB-2 is not a major factor in the development of colorectal cancer. $\mathrm{Br} \mathrm{J}$ Cancer, 86, 568-73.

Mishra K, Behari A, Kapoor VK, et al (2013). Vascular endothelial growth factor single nucleotide polymorphism in gall bladder cancer. J Gastroenterol Hepatol, 28, 1678-85.

Muddana V, Park J, Lamb J, et al (2010). Are genetic variants in the platelet-derived growth factor [beta] gene associated with chronic pancreatitis? Pancreas, 39, 1215-9.

Nakajima M, Sawada H, Yamada Y, et al (1999). The prognostic significance of amplification and overexpression of c-met and c-erb B-2 in human gastric carcinomas. Cancer, 85 , 1894-902.

Nakazawa K, Dobashi Y, Suzuki S, et al (2005). Amplification and overexpression of c-erbB-2, epidermal growth factor receptor and c-met in biliary tract cancers. J Pathol, 206, 356-65.

National Cancer Registry Programme (2002). Two-year report of the Population Based Cancer Registries, 1997-98. New Delhi: Indian Council of medical research.

Panagiotou I, Georgiannos SN, Tsiambas E, et al (2012). Impact of HER2 and PTEN simultaneous deregulation in non-small cell lung carcinoma: correlation with biological behavior. 
Kumudesh Mishra et al

Asian Pac J Cancer Prev, 13, 6311-8.

Papewalis J, Nikitin AYu, Rajewsky MF (1991). G to A polymorphism at amino acid codon 655 of the human erbB-2/ HER2gene. Nucleic Acids Res, 19, 5452.

Prakash S, Prasad N, Sharma RK, Faridi RM, Agrawal S (2012). Vascular endothelial growth factor gene polymorphisms in North Indian patients with end stage renal disease. Cytokine, 58, 261-6.

Qu K, Liu SN, Chang HL, et al (2012). Gallbladder cancer: a subtype of biliary tract cancer which is a current challenge in China. Asian Pac J Cancer Prev, 13, 1317-20.

Quaye L, Song H, Ramus SJ, et al (2009). Tagging singlenucleotide polymorphisms in candidate oncogenes and susceptibility to ovarian cancer. Br J Cancer, 100,993-1001.

Rakhshani N, Kalantari E, Bakhti H, Sohrabi MR, Mehrazma M (2014). Evaluation of HER-2/neu overexpression in gastric carcinoma using a tissue microarray. Asian Pac J Cancer Prev, 15, 7597-602.

Randi G, Franceschi S, La Vecchia C (2006). Gallbladder cancer worldwide: geographi-cal distribution and risk factors. Int J Cancer, 118, 1591-602.

Solé X, Guinó E, Valls J, Iniesta R, Moreno V (2006). SNPStats: a web tool for the analysis of association studies. Bioinformatics, 22, 1928-9.

Wang L, Habuchi T, Takahashi T, et al (2002). No association between $H E R 2$ gene polymorphism at codon 655 and a risk of bladder cancer. Int J Cancer, 97, 787-90.

Warde-Farley D, Donaldson SL, Comes O, et al (2010). The GeneMANIA prediction server: biological network integration for gene prioritization and predicting gene function. Nucleic Acids Res, 38, 214-20.

Wong FH, Hu CP, Chiu JH, et al (1994). Expression of multiple oncogenes in human esophageal carcinomas. Cancer Invest, 12, 121-31.

Xie D, Shu XO, Deng Z, et al (2000). Population-based, casecontrol study of HER2 genetic polymorphism and breast cancer risk. J Natl Cancer Inst, 92, 412-7.

Yamamoto Y, Yamagishi S, Hsu CC, Yamamoto H (1996). Advanced glycation end products-receptor interactions stimulate the growth of human pancreatic cancer cells through the induction of platelet-derived growth factor-B. Biochem Biophys Res Commun, 222, 700-5.

Zahir ST, Tafti HF, Rahmani K (2014). Overexpression of HER$2 /$ neu in patients with prostatic adenocarcinoma. Asian Pac J Cancer Prev, 15, 6425-8. 